\title{
Non-balanced translocation between the short arms of chromosomes 8 and 6 associated with type 1 diabetes mellitus
}

\author{
Vitor Scalone Netto[1]1, Gabriel Bellincanta1, Guido de Paula Colares Neto(101,2, \\ Nara Michelle de Araujo Evangelista (D2), Carolina Costa Figueiredo(D2, \\ Patricia Salmona (1D)3 and Vânia de Fátima Tonetto-Fernandes(iD),2 \\ ${ }^{1}$ Faculty of Medicine, Centro Universitário São Camilo, São Paulo, São Paulo, Brasil, 2Department of Pediatric \\ Endocrinology, Hospital Infantil Darcy Vargas, São Paulo, São Paulo, Brasil, and ${ }^{3}$ Department of Pediatric Genetics, \\ Hospital Infantil Darcy Vargas, São Paulo, São Paulo, Brasil
}

\author{
Correspondence \\ should be addressed \\ to Vânia de Fátima \\ Tonetto-Fernandes \\ Email \\ vaniatonetto@uol.com.br
}

\section{Summary}

We describe a rare case of a girl with an initial diagnostic hypothesis of chromosome 8 trisomy based on clinical findings and karyotyping, which identified a structural change in the short arm of chromosome $8(46, \mathrm{XX}$, add (8)(p23)). At the age of 7, she developed type 1 diabetes mellitus and started insulin therapy with multiple daily doses, and then she started to use a continuous insulin infusion system (pump) at 10 years of age. At the age of 12 , she underwent a molecular study that identified an unbalanced translocation between the short arms of chromosomes 6 and $8-46, X X$,add(8)(p23).ish der(8) $\mathrm{t}(6 ; 8)(\mathrm{GS}-19615+; \mathrm{RP}-11338 \mathrm{~B} 22-)$.

\section{Learning points:}

- Patients with an unbalanced translocation between the short arms of chromosomes 6 and $8-46, X X$, add(8)(p23). ish der(8)t(6;8)(GS-19615+;RP-11338B22-) may present syndromic features suggestive of chromosome 8 trisomy.

- Main characteristics are a prominent forehead, ocular and breast hypertelorism, ocular, external ear and palate abnormalities, a short neck, heart defects, and developmental delay.

- Patients with 46,XX,add(8)(p23).ish der(8)t(6;8)(GS-196I5+;RP-11338B22-) may present autoimmune type 1 diabetes mellitus.

- Karyotyping is an essential tool for the diagnosis of chromosomal changes, but it has some limitations.

- Multiplex ligation-dependent probe amplification, array-single nucleotide polymorphism and fluorescence in situ hybridization can help diagnose genetic syndromes in patients with atypical evolution.

\section{Background}

Since the advancement of molecular studies, many clinical diagnoses have been reviewed and redefined, providing a dazzling new prognostic horizon for some patients. We describe a girl who received an initial diagnosis of mosaic chromosome 8 trisomy or Warkany syndrome in the present case. The prevalence of this syndrome is estimated to be from 1:25000 to 1:50 000 live births
(1), with a male-to-female ratio of 5:1 (2). There is wide phenotypic variability among patients with mosaicism, but some manifestations are common between cases involving translocations of chromosomes 6 and 8, such as ophthalmic, digital, and CNS abnormalities. This study aimed to report a rare case of a 12-year-old girl diagnosed with unbalanced translocation between the short arms 
of chromosomes 6 and $8-46, \mathrm{XX}$,add(8)(p23).ish $\operatorname{der}(8)$ $\mathrm{t}(6 ; 8)$ (GS-19615+;RP-11338B22-) - that evolved with autoimmune type 1 diabetes mellitus. To our knowledge, this association has not yet been described in the literature.

\section{Case presentation}

The patient was the second daughter of ethnic nonconsanguineous parents and was born when her mother was 27 years old and her father was 31 years old; the mother had no previous history of abortions. Both parents denied the use of drugs, alcoholism, smoking, chronic diseases, and genetic syndromes. The child's prenatal care was adequate and uneventful. She was born by cesarean delivery due to fetal distress at 40 weeks of gestational age; her weight, length, and head circumference at birth were $2.550 \mathrm{~g}, 46$ $\mathrm{cm}$, and $31 \mathrm{~cm}$, respectively. She had a lower central incisor tooth. She required mechanical ventilation and support in a neonatal intensive care unit for 23 days, but the neonatal screening test showed no changes. Echocardiogram showed interatrial communication of $3 \mathrm{~mm}$ with shunt from left to right, without hemodynamic repercussions but with a spontaneous resolution at 4 years of age. Ultrasonography of the kidneys and urinary tract registered a slight pyelocaliceal dilation on the left. The patient evolved with delayed neuropsychomotor development. She began walking at 3.5 years of age and started intelligible speech and sphincter control at 5 years of age. Nuclear MRI of the skull revealed basilar invagination with effacement of the cerebrospinal fluid transition spaces. At 7 years of age, the patient was diagnosed with autoimmune type 1 diabetes mellitus ketoacidosis with positive anti-GAD (1191.5 UI/L; reference value: $<10 \mathrm{UI} / \mathrm{L}$ ) and anti-insulin antibodies (17.7 UI/L; reference value: <10 UI/L). After metabolic compensation, she started insulin therapy with multiple daily doses (insulins glargine and lispro) combined with carbohydrate counting and a glucose monitoring flash system. To maintain adequate glycemic control, especially during puberty, she started to use a continuous insulin infusion system (pump) at 10 years of age with suitable adaptation. Thus, she achieved adequate glycemic control with glycated hemoglobin levels between 6.0 and $6.7 \%$. Currently, the patient is 12 years old and eutrophic ( $Z$ score of the BMI-0.02). She has a short stature ( $Z$ score of height -2.37 ) and physiological evolution of puberty with recent menarche. Therefore, she has normal hearing, a moderate language disorder, bilateral astigmatism, and a normal fundus. Regarding the phenotype, the main characteristics found are described in Fig. 1.

\section{Investigation}

The karyotype was determined using the conventional G-band technique, which identified a structural alteration in the short arm of chromosome 8 [46, XX, add (8) (p23)] that was not found in the other family members (Fig. 2).

The patient's multiplex ligation-dependent probe amplification (MLPA) exam identified the deletion of the
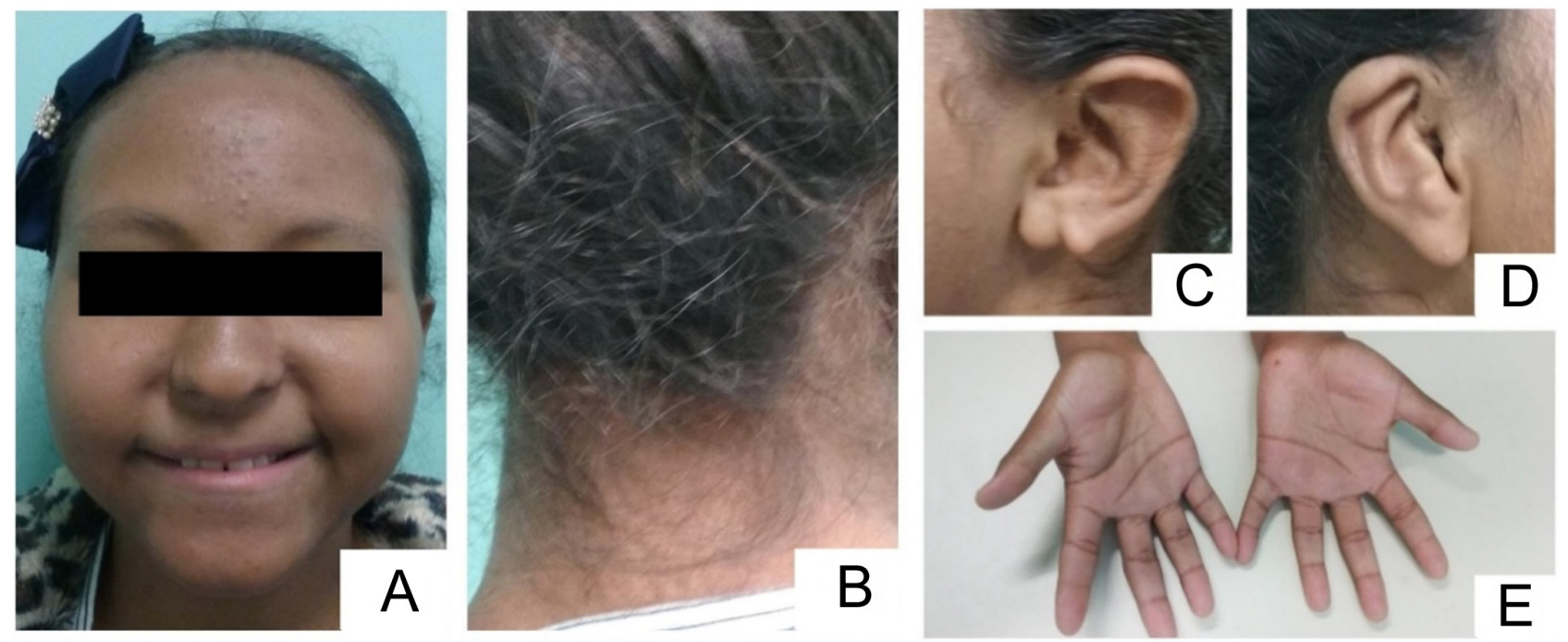

\section{Figure 1}

(A) Facial dysmorphism, prominent forehead with bulging frontal, hypertelorism, micrognathia, thin upper lip, enlarged nasal base, and nasolabial groove asymmetry; (B) Low hair implantation; (C) Bifid left ear lobe and enlarged ear shell; (D) Hyperwinding of the upper edge of the right ear helix; (E) Left hypoplasia, deep palmar grooves, small clinodactyly, and the second phalanx of the 5th finger was reduced. 

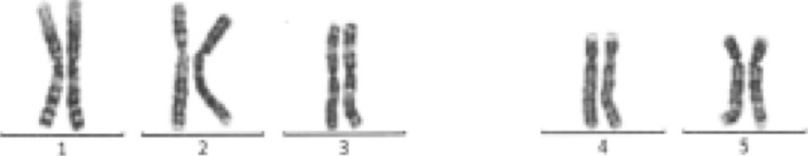

8
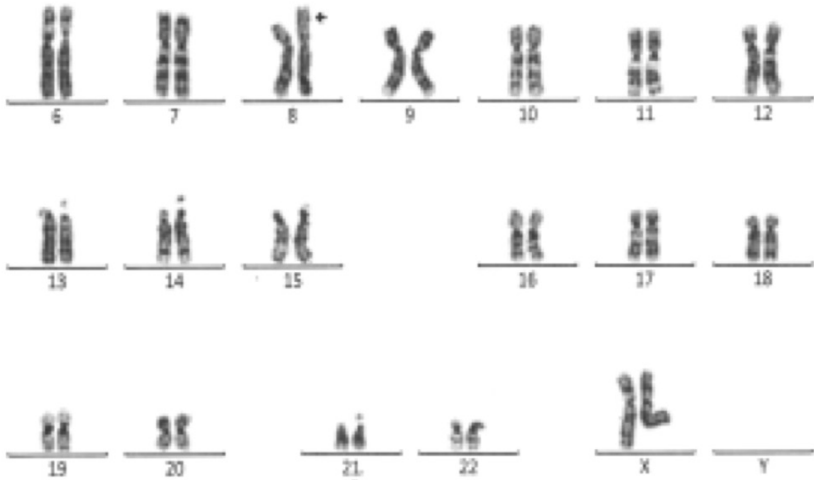

Figure 2

G-banding karyotype, peripheral blood: 46, XX, add (8) (p23) shows additional material of unknown origin on the short arm of one of chromosomes 8 in 8 p23, from 400 to 550 bands. Karyotype of parents: normal; $46, \mathrm{XX}$ and $46, \mathrm{XY}$.

terminal segment of the short arm of chromosome 8 (8p) and the duplication of the terminal segment of the short arm of chromosome 6 (6p).

The genomic DNA was examined by array-single nucleotide polymorphism (array-SNP) using the Illumina Infinium CytoSNP 850k platform and software BlueFuse Multi (Illumina). The genome version used was GRCh38; target regions have high coverage density with one probe for every $1 \mathrm{~Kb}$, while non-genic regions (backbone) have one probe for every $5 \mathrm{~kb}$. It identified 2-terminal chromosomal alterations, both classified as pathogenic: one a $25 \mathrm{Mb}$ segment duplication of the short arm of chromosome 6, arr[GRCh38] 6p25.3p22.3(108666_25065441)x3, and a $3.4 \mathrm{Mb}$ segment deletion of the short arm of chromosome 8, $\operatorname{arr}[$ GRCh38] 8p23.3p23.2(214984_3663401)x1.

Fluorescence in situ hybridization (FISH) analysis performed on the patient's sample showed a single signal from probe RP11-338B22 (8p23.3) on one chromosome 8 and three signals from probe GS-196I5 (6p25.3), one signal on one chromosome 8 and the other two signals on chromosome pair 6 . This result agrees with the MLPA test findings and confirms the presence of unbalanced translocation between the short arms of chromosomes 8 and 6 (Fig. 3). According to the expected pattern, analysis of the parent's sample showed two signals from the RP11338B22 probe on chromosome pair 8 and two signals from

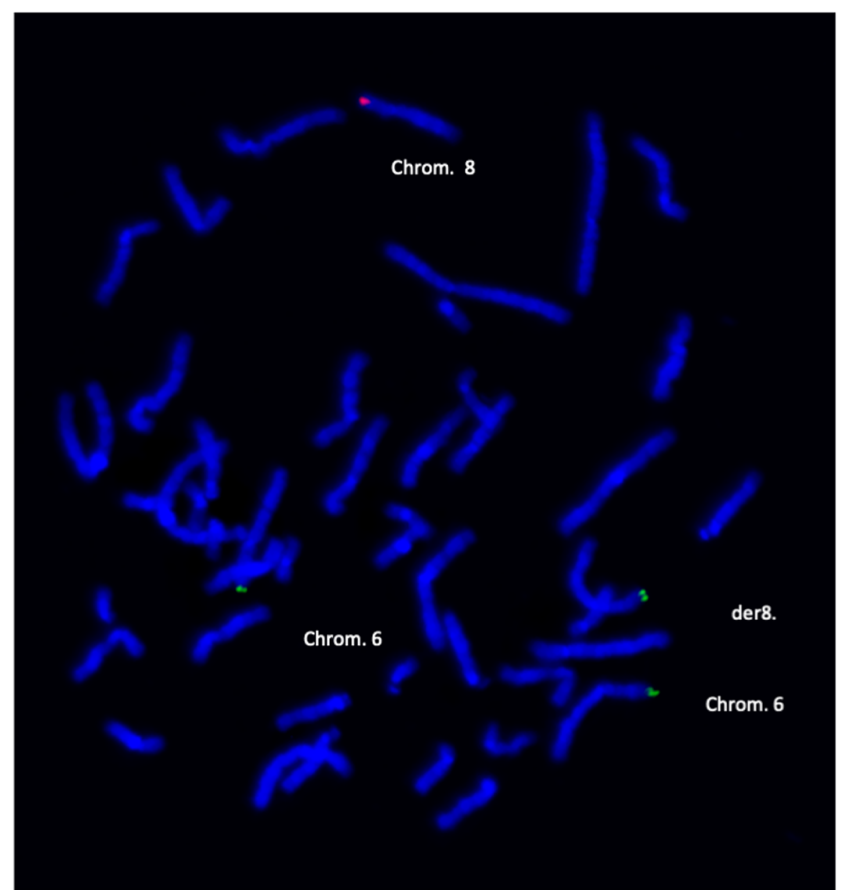

Figure 3

Fluorescence in situ hybridization was performed with probes that map in the terminal regions of the short arm of chromosome 6 (GS196I5 -

marked with biotin and visualized with FITC - green) and of the short arm of chromosome 8 (RP11338B22 - marked with digoxigenin and visualized with rhodamine - red).

the probe GS-196I5 on chromosome pair 6, indicating that the chromosomal changes identified in the daughter were due to a new mutation.

\section{Treatment}

Currently, the patient uses the insulin pump with $56 \%$ of basal insulin and $44 \%$ of bolus insulin. In every meal, she counts carbohydrates that vary between 1:7 and 1:25 g. Her sensitivity factor for insulin bolus is 90 during the day and 110 during the night.

\section{Outcome and follow-up}

A continuous insulin infusion system (pump) proved helpful as the patient continues with adequate glycemic control. Nowadays, her glycated hemoglobin is 5.6\%, and her glucose monitoring was $91 \%$ adequate, $6 \%$ of hyperglycemia, and 3\% of hypoglycemia. Clinical improvement in neurodevelopment is maintained with regular psychological, psycho-pedagogical, and speech therapies. 


\section{Discussion}

We describe a case of a girl with syndromic features suggestive of chromosome 8 trisomy. Reports related to this genetic alteration indicate that the main characteristics are a prominent forehead, ocular and breast hypertelorism, ocular and external ear abnormalities, abnormalities of the palate, a short neck, defects leading to heart failure, mental retardation, and developmental delay (3). The patient in question presents 17 of the $27(62,9 \%)$ clinical aspects described in the literature of cases with alterations detected in partial monosomy of $8 p$ and partial trisomy of $6 \mathrm{p}$ (Table 1) $(4,5)$. She had this diagnosis from 8 months to 12 years of age when she participated in this study, and 46,XX, add(8)(p23).ish $\operatorname{der}(8) \mathrm{t}(6 ; 8) \quad$ (GS-196I5+;RP11338B22-) was identified.

Although karyotyping is an essential tool for the diagnosis of chromosomal changes, it has some limitations, which are mainly linked to the increase

Table 1 Comparison of the clinical findings in patients with partial trisomy of the short arm of chromosome 6 and partial monosomy of the long arm of chromosome 8 in previous case reports with the patient reported.

\begin{tabular}{|c|c|c|c|}
\hline Clinical findings & $\begin{array}{l}\text { 6pter } \\
\text { trisomy }\end{array}$ & $\begin{array}{c}\text { 8pter } \\
\text { monosomy }\end{array}$ & Patient \\
\hline Low birth weight & + & - & - \\
\hline Short stature & + & + & + \\
\hline Microcephaly & + & + & - \\
\hline Prominent forehead & + & - & + \\
\hline Ocular hypertelorism & - & - & + \\
\hline Abnormal eyelid cleft & + & - & + \\
\hline Eye abnormalities & + & + & + \\
\hline Flat/wide nasal base & + & + & + \\
\hline Elevated nasal tip & - & - & - \\
\hline External ear abnormalities & + & + & + \\
\hline Hearing loss & + & - & - \\
\hline Anomalies of the palate & + & - & + \\
\hline Small mouth & + & + & + \\
\hline Micrognathia & + & + & + \\
\hline Small chin & + & - & - \\
\hline Short neck & + & + & + \\
\hline Heart defects & + & + & + \\
\hline Pectus deformity & - & + & - \\
\hline Clinodactyly & + & - & + \\
\hline Digital anomalies & + & - & + \\
\hline Large fontanel and sutures & + & - & - \\
\hline CNS malformations & + & + & + \\
\hline Psychomotor retardation & + & + & + \\
\hline Developmental delay & + & + & + \\
\hline Genito-urinary anomalies & + & + & - \\
\hline Sacral dimple & + & - & - \\
\hline Vertebral anomalies & - & + & - \\
\hline
\end{tabular}

Adapted from Castiglione (4) and Papadopoulou (5).

+: present; -: absent. in band resolution. Thus, more sensitive and accurate techniques such as next-generation sequencing, MLPA, FISH, and array-SNP can help diagnose genetic syndromes in patients with atypical evolution represented by the case in question.

In patients with $46, \mathrm{XX}, \mathrm{add}(8)(\mathrm{p} 23)$.ish $\operatorname{der}(8) \mathrm{t}(6 ; 8)$ (GS-19615+;RP-11338B22-), the association with type 1 diabetes mellitus is very rare. In some chromosopathies, such as trisomy 21 , the prevalence of autoimmune diseases such as diabetes has been higher than that in the general population (6). They evolve with better glycemic control when an insulin pump is included in the treatment protocol, which was also observed in this case $(7,8,9)$.

In conclusion, our study reports the case of a patient diagnosed with 46,XX, add(8)(p23).ish $\operatorname{der}(8) \mathrm{t}(6 ; 8)$ (GS-196I5+;RP-11338B22-) who developed autoimmune type 1 diabetes mellitus. We highlight the importance of molecular studies for elucidating the accurate diagnosis of genetic changes without initially defined etiologies and detecting metabolic disorders that may be present in rare diseases, indicating an association with acquired pathologies.

\section{Patient's perspective}

Although the disease's diagnosis was challenging, the patient's mother refers that the multidisciplinary treatment was essential for managing her daughter's disease. Also, she refers to improving the family's quality of life after the insulin pump's use. Due to better glycemic control, the child could go to school regularly and maintain her social activities.

\section{Declaration of interest}

The authors declare that there is no conflict of interest that could be perceived as prejudicing the impartiality of the research reported.

\section{Funding}

This study did not receive any specific grant from any funding agency in the public, commercial or not-for-profit sector.

\section{Patient consent}

This study was conducted with the approval of the Ethics Committee of Hospital Infantil Darcy Vargas. Written informed consent for publication of the submitted article and accompanying images was obtained from the patient's parents, following the Declaration of Helsinki's ethical guidelines established in 2008.

\section{Author contribution statement}

Vitor Scalone Netto and Gabriel Bellincanta were responsible for collecting data from the patient and writing this article. Guido de Paula Colares Neto, Nara Michelle de Araujo Evangelista and Carolina Costa Figueiredo were 
responsible for type 1 diabetes mellitus follow-up. Patricia Salmona was responsible for genetic exam analysis. Vânia de Fátima Tonetto-Fernandes was the endocrinologist in charge of the patient and was also responsible for the article's review.

\section{Acknowledgements}

The authors are grateful to biologists Dr Carla Rosenberg and Dr Ana Krepischi from the Human Genome and Stem Cell Research Center and Dr Celia Priszkulnik Koiffmann - Genetic Counseling Unit of the Department of Genetics and Evolutionary Biology - University of São Paulo. Also, the authors are thankful to the patient's parents for their collaboration.

\section{References}

1 Wiśniewska M \& Mazurek M. Trisomy 8 mosaicism syndrome. Journal of Applied Genetics 200243 115-118.(https://www.researchgate.net/ publication/11290367_Trisomy_8_mosaicism_syndrome )

2 Udayakumar AM \& Al-Kindy A. Constitutional trisomy 8 mosaicism syndrome: case report and review. Journal of Pediatric Genetics 20132 197-201. (https://doi.org/10.3233/PGE-13069)

3 Jones KL. Smith's Recognizable Patterns of Human Malformation, 7th ed., pp. 24-28. Philadelphia: Saunders, 2013.

4 Castiglione A, Guaran V, Astolfi L, Orioli E, Zeri G, Gemmati D, Bovo R, Montaldi A, Alghisi A \& Martini A. Karyotype-phenotype correlation in partial trisomies of the short arm of chromosome 6: a family case report and review of the literature. Cytogenetic and Genome Research 2013141 243-259.(https://doi.org/10.1159/000353846)

5 Papadopoulou Z, Papoulidis I, Sifakis S, Markopoulos G, Vetro A, Vlaikou AM, Ziegler M, Liehr T, Thomaidis L, Zuffardi O, et al. Partial monosomy $8 \mathrm{p}$ and trisomy $16 \mathrm{q}$ in two children with developmental delay detected by array comparative genomic hybridization. Molecular Medicine Reports 201716 8808-8818. (https://doi.org/10.3892/ mmr.2017.7760)

6 Anwar AJ, Walker JD \& Frier BM. Type 1 diabetes mellitus and Down's syndrome: prevalence, management and diabetic complications. Diabetic Medicine 199815 160-163. (https://doi.org/10.1002/ (SICI)1096-9136(199802)15:2<160::AID-DIA537>3.0.CO;2-J)

7 Hawli Y, Nasrallah M \& Fuleihan GH. Endocrine and musculoskeletal abnormalities in patients with Down syndrome. Nature Reviews: Endocrinology 20095 327-334. (https://doi.org/10.1038/ nrendo.2009.80)

8 Rohrer TR, Hennes P, Thon A, Dost A, Grabert M, Rami B, Wiegand S, Holl RW \& DPV Initiative. Down's syndrome in diabetic patients aged <20 years: an analysis of metabolic status, glycaemic control and autoimmunity in comparison with type 1 diabetes. Diabetologia 201053 1070-1075. (https://doi.org/10.1007/s00125-0101686-z)

9 Scaramuzza AE, Giani E, Riboni S, Spiri D, De Palma A, Mameli C \& Zuccotti GV. Insulin pump therapy for type 1 diabetes treatment in a girl with Down's syndrome. Diabetes Research and Clinical Practice 2009 85 e16-e18. (https://doi.org/10.1016/j.diabres.2009.06.008)

Received in final form 29 August 2021

Accepted 25 October 2021 